CUBO A Mathematical Journal

Vol.21, № 03, (63-74). December 2019

\title{
Beta-almost Ricci solitons on Sasakian 3-manifolds
}

\author{
Pradip Majhi and Debabrata Kar \\ Department of Pure Mathematics, \\ University of Calcutta, \\ 35, Ballygaunge Circular Road, \\ Kolkata 700019, West Bengal, India \\ mpradipmajhi@gmail.com, debabratakar6@gmail.com
}

\begin{abstract}
In this paper we characterize the Sasakian 3-manifolds admitting $\beta$-almost Ricci solitons whose potential vector field is a contact vector field. Among others we prove that a $\beta$ almost Ricci soliton whose potential vector field is a contact vector field on a Sasakian 3-manifold is shrinking, Einstein and non-trivial. Moreover, we prove that this type of manifolds are isometric to a sphere of radius $\sqrt{7}$.
\end{abstract}

\section{RESUMEN}

En este artículo caracterizamos las 3 -variedades Sasakianas que admiten solitones $\beta$ casi Ricci cuyo campo de vectores potencial es un campo de vectores de contacto. Entre otros, probamos que un solitón $\beta$-casi Ricci cuyo campo de vectores potencial es un campo de vectores de contacto en una 3-variedad Sasakiana se contrae, es Einstein y no trivial. Más aún, probamos que este tipo de variedades son isométricas a una esfera de radio $\sqrt{7}$.

Keywords and Phrases: Ricci soliton, $\beta$-almost Ricci soliton, Sasakian 3-manifolds, Einstein. 2010 AMS Mathematics Subject Classification: 53C15, 53C25. 


\section{Introduction}

In 1982, R. S. Hamilton [17 introduced the notion of Ricci flow to find a canonical metric on a smooth manifold. The Ricci flow is an evolution equation for metrics on a Riemannian manifold defined as follows:

$$
\frac{\partial}{\partial t} g=-2 S
$$

where $\mathrm{S}$ denotes the Ricci tensor of $\mathrm{g}$. Ricci solitons are special solutions of the Ricci flow equation (11) of the form $g=\sigma(t) \psi_{t}^{*} g$ with the initial condition $g(0)=g$, where $\psi_{t}$ are diffeomorphisms of $M$ and $\sigma(t)$ is the scaling function. A Ricci soliton is a generalization of an Einstein metric. We recall the notion of Ricci soliton according to [5]. On the manifold $M$, a Ricci soliton is a triple $(g, V, \lambda)$ with $g$, a Riemannian metric, $V$ a vector field, called the potential vector field and $\lambda$ a real scalar such that

$$
£ v g+2 S+2 \lambda g=0
$$

where $£$ is the Lie derivative. Metrics satisfying (2) are interesting and useful in physics and are often referred as quasi-Einstein $([6,[7])$. Compact Ricci solitons are the fixed points of the Ricci flow $\frac{\partial}{\partial t} g=-2 S$ projected from the space of metrics onto its quotient modulo diffeomorphisms and scalings, and often arise blow-up limits for the Ricci flow on compact manifolds. Theoretical physicists have also been looking into the equation of Ricci soliton in relation with string theory. The initial contribution in this direction is due to Friedan [14] who discusses some aspects of it. Recently, the notion of almost Ricci soliton has been introduced in 24] by Piagoli, Riogoli, Rimoldi and Setti.

The Ricci soliton is said to be shrinking, steady or expanding according as $\lambda$ is negative, zero or positive respectively. Ricci solitons have been studied by several authors ([8], [9, [18, [19], [20], [27, [28, and many others).

Recently, Gomes, Wang and Xia [26] generalized almost Ricci soliton to $h$-almost Ricci soliton as follows:

Definition 1.1. A complete connected Riemannian manifold $\left(M^{2 n+1}, g\right)$ is said to be a $\beta$-almost Ricci soliton, denoted by $\left(\mathrm{M}^{2 \mathrm{n}+1}, \mathrm{~g}, \mathrm{~V}, \beta, \lambda\right)$, if there exist a smooth vector field $\mathrm{V}$ on $\mathrm{M}^{2 \mathrm{n}+1}$ such that

$$
\mathrm{S}+\frac{\beta}{2} £ \mathrm{vg}+\lambda \mathrm{g}=0
$$

where $\lambda$ and $\beta$ are smooth functions on $\mathrm{M}^{2 \mathrm{n}+1} . \lambda$ is called soliton function and $\mathrm{V}$ is called the potential vector field. 
A $\beta$-almost Ricci soliton is said to be shrinking, steady or expanding according as $\lambda$ is negative, zero or positive respectively. A $\beta$-almost Ricci soliton is called $\beta$-Ricci soliton if $\lambda$ is constant. A $\beta$-almost Ricci soliton is said to be trivial, that is, Einstein if the flow vector field $V$ is homothetic, that is, $£_{V} g=c g$, for some constant c. Otherwise, it is called non-trivial. A $\beta$-almost Ricci soliton is said to be $\beta$-almost gradient Ricci soliton if the potential vector field $\mathrm{V}$ is the gradient of a smooth function $f$ on $M^{2 n+1}$, that is, $V=D f$, where $D$ is the gradient operator of $g$ on $M^{2 n+1}$.

For convenience, we denote $\left(M^{2 n+1}, g, D f, \beta, \lambda\right)$ as a $\beta$-almost gradient Ricci soliton with potential function $f$.

In particular, a Ricci soliton is a 1-almost Ricci soliton with constant soliton $\lambda$ and an almost Ricci soliton is nothing but a 1-almost Ricci soliton. Recently, Ghosh and Patra studied [16] the k-almost Ricci solitons on contact geometry. In [1], Barros and Ribeiro proved that a compact almost Ricci soliton with constant scalar curvature is isometric to an Euclidean sphere. In this connection, a theorem has also been proved by Gomes, Wang and Xia in [26] for $\beta$-almost Ricci soliton which is given as follows:

Theorem 1.1. [26] Let $\left(\mathrm{M}^{\mathrm{n}}, \mathrm{g}, \mathrm{V}, \beta, \lambda\right), \mathrm{n} \geq 3$ be a non-trivial $\beta$-almost Ricci soliton with constant scalar curvature $\mathrm{r}$. If $\mathrm{M}^{\mathrm{n}}$ is compact, then it is isometric to a standard sphere $\mathrm{S}^{\mathrm{n}}$ (c) of radius $c=\sqrt{\frac{2 n(2 n+1)}{r}}$.

The above Theorem will be used in later to prove our results.

The paper is organized as follows:

After preliminaries in Section 2, we study $\beta$-almost Ricci solitons on a Sasakian 3-manifold. Among others we prove that $\beta$-almost Ricci solitons whose potential vector field is a contact vector field on Sasakian 3-manifolds are shrinking and Einstein. Beside these, we prove that this type of manifolds are isometric to a sphere of radius $\sqrt{7}$. Also we prove that a $\beta$-almost Ricci soliton whose potential vector field is a contact vector field on a Sasakian 3-manifold is non-trivial.

\section{Preliminaries}

An odd dimensional smooth manifold $M^{2 n+1}(n \geq 1)$ is said to admit an almost contact structure, sometimes called a $(\phi, \xi, \eta)$-structure, if it admits a tensor field $\phi$ of type $(1,1)$, a vector field $\xi$ and a 1 -form $\eta$ satisfying ([2], [3])

$$
\phi^{2}=-\mathrm{I}+\eta \otimes \xi, \eta(\xi)=1, \quad \phi \xi=0, \eta \circ \phi=0 .
$$


The first and one of the remaining three relations in (44) imply the other two relations in (44). An almost contact structure is said to be normal if the induced almost complex structure $J$ on $M^{\mathfrak{n}} \times \mathbb{R}$ defined by

$$
J\left(X, f \frac{d}{d t}\right)=\left(\phi X-f \xi, \eta(X) \frac{d}{d t}\right)
$$

is integrable, where $X$ is tangent to $M, t$ is the coordinate of $\mathbb{R}$ and $f$ is a smooth function on $M^{n} \times \mathbb{R}$. Let $g$ be a compatible Riemannian metric with the $(\phi, \xi, \eta)$-structure, that is,

$$
g(\phi X, \phi Y)=g(X, Y)-\eta(X) \eta(Y)
$$

or equivalently,

$$
g(X, \phi Y)=-g(\phi X, Y)
$$

and

$$
g(X, \xi)=\eta(X),
$$

for all vector fields $X, Y$ tangent to $M$. Then $M$ becomes an almost contact metric manifold equipped with an almost contact metric structure $(\phi, \xi, \eta, g)$.

An almost contact metric structure becomes a contact metric structure if

$$
g(X, \phi Y)=d \eta(X, Y)
$$

for all $X, Y$ tangent to $M$. The 1 -form $\eta$ is then a contact form and $\xi$ is its characteristic vector field.

Given the contact metric manifold $(M, \eta, \xi, \phi, g)$, we define a symmetric $(1,1)$-tensor field $h$ as $\mathrm{h}=\frac{1}{2} \mathrm{~L}_{\xi} \phi$, where $\mathrm{L}_{\xi} \phi$ denotes Lie differentiation in the direction of $\xi$. We have the following identities $([2],[3])$ :

$$
\begin{gathered}
h \xi=0, \quad h \phi+\phi h=0, \\
\nabla_{X} \xi=-\phi X-\phi h X \\
\nabla_{\xi} \phi=0, \\
R(\xi, X) \xi-\phi R(\xi, \phi X) \xi=2\left(h^{2}+\phi^{2}\right) X \\
\left(\nabla_{\xi} h\right) X=\phi X-h^{2} \phi X+\phi R(\xi, X) \xi \\
S(\xi, \xi)=2 n-\operatorname{trh}^{2}, \\
R(X, Y) \xi=-\left(\nabla_{X} \phi\right) Y+\left(\nabla_{Y} \phi\right) X-\left(\nabla_{X} \phi h\right) Y+\left(\nabla_{Y} \phi h\right) X .
\end{gathered}
$$

Here, $\nabla$ is the Levi-Civita connection and $R$ is the Riemannian curvature tensor of $(M, g)$ with the sign convention defined by

$$
\mathrm{R}(\mathrm{X}, \mathrm{Y}) \mathrm{Z}=\nabla_{\mathrm{X}} \nabla_{\mathrm{Y}} \mathrm{Z}-\nabla_{\mathrm{Y}} \nabla_{\mathrm{X}} \mathrm{Z}-\nabla_{[\mathrm{X}, \mathrm{Y}]} \mathrm{Z}
$$


for vector fields $X, Y, Z$ on $M$. The tensor $l=R(., \xi) \xi$ is the Jacobi operator with respect to the characteristic field $\xi$.

If the characteristic vector field $\xi$ is a Killing vector field, the contact metric manifold $(M, \eta, \xi, \phi, g)$ is called $\mathrm{K}$-contact manifold. This is the case if and only if $h=0$. The contact structure on $M$ is said to be normal if the almost complex structure on $M \times \mathbb{R}$ defined by (5), is integrable. A normal contact metric manifold is called a Sasakian manifold. Sasakian metrices are K-contact and K-contact metrics on 3-manifolds are Sasakian. For a Sasakian manifold, the following hold $([2],[3])$ :

$$
\begin{gathered}
\nabla_{X} \xi=-\phi X \\
\left(\nabla_{X} \phi\right) Y=g(X, Y) \xi-\eta(Y) X, \\
R(X, Y) \xi=\eta(Y) X-\eta(X) Y, \\
Q \xi=2 n \xi,
\end{gathered}
$$

where $\mathrm{Q}$ denotes the $(1,1)$-tensor metrically equivalent to the Ricci tensor of $\mathrm{g}$. The curvature tensor of a 3-dimensional Riemannian manifold is given by

$$
\begin{aligned}
R(X, Y) Z= & {[S(Y, Z) X-S(X, Z) Y+g(Y, Z) Q X-g(X, Z) Q Y] } \\
& -\frac{r}{2}[g(Y, Z) X-g(X, Z) Y],
\end{aligned}
$$

where $S$ and $r$ are the Ricci tensor and scalar curvature respectively and $Q$ is the Ricci operator defined by $g(Q X, Y)=S(X, Y)$.

It is known that the Ricci tensor of a Sasakian 3-manifold is given by [4]

$$
S(X, Y)=\frac{1}{2}\{(r-2) g(X, Y)+(6-r) \eta(X) \eta(Y)\}
$$

where $r$ is the scalar curvature which need not be constant, in general. So, $g$ is Einstein (hence has constant curvature 1 ) if and only if $r=6$.

As a consequence of (23), we have

$$
S(X, \xi)=2 \eta(X)
$$

Contact metric manifolds have also been studied by several authors (44, 10], 11], 12, 13], [21, [22, 23], 25], and many others). 
Definition 2.1. ([16]) A vector field $\mathrm{V}$ on a contact manifold is said to be a contact vector field if it preserves the contact form $\eta$, that is

$$
£_{\vee \eta}=\psi \eta
$$

for some smooth function $\psi$ on $\mathrm{M}$. When $\psi=0$ on $\mathrm{M}$, the vector field $\mathrm{V}$ is called a strict contact vector field.

Lemma 2.1. ([15]) If a vector field $X$ leaves the structure tensor $\phi$ of the contact metric manifold $M$ invariant, then there exists a constant $\mathrm{c}$ such that $£_{\mathrm{X}} \mathrm{g}=\mathrm{c}(\mathrm{g}+\eta \otimes \eta)$.

\section{$3 \quad \beta$-almost Ricci solitons on Sasakian 3-manifolds}

In this section we characterize Sasakian 3-manifolds $M^{3}$ admitting $\beta$-almost Ricci solitons whose potential vector field $V$ is a contact vector field. Then the equations (3) and (25) hold good. The equation (3) can be exhibited as

$$
S(X, Y)+\frac{\beta}{2}\left\{g\left(\nabla_{X} V, Y\right)+g\left(X, \nabla_{Y} V\right)\right\}+\lambda g(X, Y)=0
$$

Using (23) in the above equation we get

$$
\begin{aligned}
\beta\left\{g\left(\nabla_{X} V, Y\right)+g\left(X, \nabla_{Y} V\right)\right\}= & -(r+2 \lambda-2) g(X, Y) \\
& +(r-6) \eta(X) \eta(Y) .
\end{aligned}
$$

Tracing the equation (27) we obtain

$$
\beta \operatorname{div} V=-(r+3 \lambda) .
$$

With the help of (25) we have

$$
£_{V} \mathrm{~d} \eta=\mathrm{d} £_{\mathrm{V} \eta}=(\mathrm{d} \psi) \wedge \eta+\psi(\mathrm{d} \eta) .
$$

Let us consider $\omega$ as the volume form of the manifold $M^{3}$, that is,

$$
\omega=\eta \wedge d \eta \neq 0 .
$$

Taking Lie derivative of the preceding equation along the potential vector field $\mathrm{V}$ and using (25) and (29) we have $£_{V} \omega=2 \psi \omega$, and hence

$$
\operatorname{div} V=2 \psi
$$


Using the foregoing equation in (28) we infer

$$
r=-2 \psi \beta-3 \lambda .
$$

The soliton equation (3) also can be represented as

$$
S(X, Y)+\frac{\beta}{2}(£ \vee g)(X, Y)+\lambda g(X, Y)=0 .
$$

Substituting $X=Y=\xi$ in (33) we get

$$
\beta g(£ \vee \xi, \xi)=\lambda+2 .
$$

Putting $Y=\xi$ in (33) and using (24),

$$
\frac{\beta}{2}(£ \vee \eta)(X)-\frac{\beta}{2} g(X, £ \vee \xi)+(\lambda+2) \eta(X)=0 .
$$

Making use of (25) we obtain

$$
\beta £ \vee \xi=(\psi \beta+2 \lambda+4) \xi .
$$

By the virtue of (34) and (36) we have

$$
\psi \beta=-\lambda-2 .
$$

Using (37), (36) entails

$$
\beta £ \mathrm{~V} \xi=(\lambda+2) \xi \text {. }
$$

From (9) we deduce that

$$
\left(£_{V} d \eta\right)(X, Y)=\left(£_{V} g\right)(X, \phi Y)+g\left(X,\left(£_{V} \phi\right) Y\right) .
$$

Multiplying both sides of (39) by $\beta$ and then using (33) we infer

$$
\beta\left(£_{V} d \eta\right)(X, Y)=-2 S(X, \phi Y)-2 \lambda g(X, \phi Y)+\beta g\left(X,\left(£_{V} \phi\right) Y\right) .
$$

In view of (23) and (40) we get

$$
\beta\left(£_{V} d \eta\right)(X, Y)=-(r+2 \lambda-2) g(X, \phi Y)+\beta g\left(X,\left(£_{V} \phi\right) Y\right) .
$$

From (29) we derive

$$
\left(£_{V} d \eta\right)(X, Y)=\frac{1}{2}\{d \psi(X) \eta(Y)-d \psi(Y) \eta(X)\}+\psi g(X, \phi Y) .
$$

Comparing (41) and (42), after simplification we obtain

$$
2 \beta\left(£_{\mathrm{V}} \phi\right) \mathrm{Y}=2(\mathrm{r}+2 \lambda-2) \phi \mathrm{Y}+\beta \eta(\mathrm{Y}) \mathrm{D} \psi-\beta(\mathrm{Y} \psi) \xi+2 \psi \beta \phi \mathrm{Y} .
$$


Replacing $Y$ by $\xi$ we get

$$
2 \beta\left(£_{V} \phi\right) \xi=\beta D \psi-\beta(\xi \psi) \xi .
$$

With the help of (4) and (36) we find that

$$
\beta\left(£_{\vee} \phi\right) \xi=0 .
$$

Applying (45) on (44) we have

$$
\mathrm{D} \psi=(\xi \psi) \xi .
$$

Taking inner product of (46) with $X$ gives

$$
\mathrm{d} \psi(X)=(\xi \psi) \eta(X),
$$

or equivalently,

$$
\mathrm{d} \psi=(\xi \psi) \eta
$$

Taking exterior derivative we get

$$
\mathrm{d}(\xi \psi) \wedge \eta+(\xi \psi) \mathrm{d} \eta=0 .
$$

Taking wedge product of (49) with $\eta$ we have

$$
(\xi \psi) \eta \wedge d \eta=0,
$$

from which it follows that

$$
\xi \psi=0,
$$

Since $\eta \wedge d \eta \neq 0$, and by [48],

$$
d \psi=0
$$

and hence $\psi$ is constant. Integrating (31) and then using Divergence Theorem we infer

$$
\psi=0 .
$$

Thus the potential vector field $\mathrm{V}$ becomes a strict contact vector field and hence we have the following:

Theorem 3.1. Let $\left(M^{3}, g, V, \beta, \lambda\right)$ be a non-trivial $\beta$-almost Ricci soliton whose potential vector field is a contact vector field on a Sasakian 3-manifold. Then the potential vector field is a strict 
contact vector field.

By the virtue of (37) and (53) we find

$$
\lambda=-2 .
$$

Therefore, the $\beta$-almost Ricci soliton is shrinking. Thus we are in a position to state that

Theorem 3.2. A non-trivial $\beta$-almost Ricci soliton $\left(M^{3}, g, V, \beta, \lambda\right)$ whose potential vector field is a contact vector field on a Sasakian 3-manifold is shrinking.

Making use of (53) and (54), from (32) we get

$$
r=6 .
$$

Then we can conclude that

Theorem 3.3. The scalar curvature of a non-trivial $\beta$-almost Ricci soliton $\left(M^{3}, g, V, \beta, \lambda\right)$ whose potential vector field is a contact vector field on a Sasakian 3-manifold is 6.

With the help of (55) from (23) we have

$$
S(X, Y)=2 g(X, Y)
$$

Hence we can state the following:

Theorem 3.4. A non-trivial $\beta$-almost Ricci soliton $\left(M^{3}, g, V, \beta, \lambda\right)$ whose potential vector field is a contact vector field on Sasakian 3-manifold is Einstein.

From (55) we can say that $r$ is constant. Then in view of Theorem 1.1 we can conclude the following:

Theorem 3.5. Let $\left(M^{3}, g, V, \beta, \lambda\right)$ be a non-trivial $\beta$-almost Ricci soliton whose potential vector field is a contact vector field on a Sasakian 3-manifolds. Then $\left(\mathrm{M}^{3}, \mathrm{~g}, \mathrm{~V}, \beta, \lambda\right)$ is isometric to a sphere $S^{3}(\mathrm{c})$ of radius $\mathrm{c}=\sqrt{7}$.

Using (53), (54) and (55) in (43) we infer

$$
(£ \vee \phi) Y=0,
$$

as we have considered $\beta$ as positive, that is, $\mathrm{V}$ leaves the structure tensor $\phi$ of the Sasakian 3-manifold invariant. Then, by Lemma 2.2, exists a constant a such that

$$
£ \vee g=a(g+\eta \otimes \eta)
$$


which shows that the $\beta$-almost Ricci solitons are non-trivial. Thus our next theorem can be stated as follows:

Theorem 3.6. Let $\left(M^{3}, g, V, \beta, \lambda\right)$ be a non-trivial $\beta$-almost Ricci soliton whose potential vector field is a contact vector field on a Sasakian 3-manifolds. Then the $\beta$-almost Ricci solitons are non-trivial.

Acknowledgement: The authors are thankful to the referee for his/her valuable suggestions and comments towards the improvement of the paper. The author Debabrata Kar is supported by the Council of Scientific and Industrial Research, India (File no : 09/028(1007)/2017-EMR-1). 


\section{References}

[1] Barros, A. and Ribeiro, Jr., Some characterizations for compact almost Ricci solitons, Proc. Amer. Math. Soc., 140(3) (2012), 1033-1040.

[2] Blair, D. E., Lecture notes in Mathematics, 509, Springer-Verlag Berlin(1976).

[3] Blair, D. E., Riemannian Geometry of contact and symplectic manifolds, Birkhäuser, Boston, 2002 .

[4] Blair, D. E., Koufogiorgos, T. and Sharma, R., A classification of 3-dimensional contact metric manifolds with $\mathrm{Q} \phi=\phi \mathrm{Q}$, Kodai Math. J., 13(1990), 391-401.

[5] Calin, C. and Crasmareanu, M., From the Eisenhart problem to Ricci solitons in f-Kenmotsu manifolds, Bull. Malays. Math. Soc., 33(3) (2010), 361-368.

[6] Chave, T. and Valent, G., Quasi-Einstein metrics and their renoirmalizability properties, Helv. Phys. Acta., 69 (1996) 344-347.

[7] Chave, T. and Valent, G., On a class of compact and non-compact quasi-Einstein metrics and their renoirmalizability properties, Nuclear Phys. B., 478 (1996), 758-778.

[8] Deshmukh, S., Jacobi-type vector fields on Ricci solitons, Bull. Math. Soc. Sci. Math. Roumanie, 55(103)1 (2012), 41-50.

[9] Deshmukh, S., Alodan, H. and Al-Sodais, H., A Note on Ricci Soliton, Balkan J. Geom. Appl. 16(1) (2011), 48-55.

[10] De, U. C. and Biswas, S., A note on $\xi$-conformally flat contact manifolds, Bull. Malays. Math. Soc., 29 (2006), 51-57.

[11] De, U. C. and Mondal, A. K., Three dimensional Quasi-Sasakian manifolds and Ricci solitons, SUT J. Math., 48(1) (2012), 71-81.

[12] De, U. C. and Mondal, A. K., The structure of some classes of 3-dimensional normal almost contact metric manifolds, Bull. Malays. Math. Soc., 36(2) (2013), 501-509.

[13] De, Avik and Jun, J. B., On N(k)-contact metric manifolds satisfying certain curvature conditions, Kyungpook Math. J., 51(4) (2011), 457-468.

[14] Friedan, D., Nonlinear models in $2+\epsilon$ dimensions, Ann. Phys., 163 (1985), 318-419.

[15] Ghosh, A. and Sharma, R., Sasakian metric as a Ricci soliton and related results, J. Geom. Phys., 75 (2014), 1-6. 
[16] Ghosh, A. and Patra, D. S., The k-almost Ricci solitons and contact geometry, J. Korean. Math., 55(1) (2018), 161-174.

[17] Hamilton, R. S., The Ricci flow on surfaces, Mathematics and general relativity, (Santa Cruz, CA, 1986), 237-262. Contemp. Math., 71, American Math. Soc., 1988.

[18] Hamilton, R. S., Three manifolds with positive Ricci curvature, J. Differential Geom., 17 (1982), 255-306

[19] Ivey, T., Ricci solitons on compact 3-manifolds, Diff. Geom. Appl., 3 (1993),301-307.

[20] Kim, B.H., Fibered Riemannian spaces with quasi-Sasakian structures, Hiroshima Math. J., 20 (1990), 477-513.

[21] Majhi, P., and De, U. C., Classifications of N(k)-contact metric manifolds satisfying certain curvature conditions, Acta Math. Univ. Commenianae, LXXXIV(1) (2015), 167-178.

[22] Özgür, C., Contact metric manifolds with cyclic-parallel Ricci tensor, Diff. Geom. Dynamical systems, 4 (2002), 21-25.

[23] Özgür, C. and Sular, S., On N(k)-contact metric manifolds satisfying certain conditions, SUT J. Math., 44(1) (2008), 89-99.

[24] Pigola, S., Rigoli, M., Rimoldi, M. and Setti, A., Ricci almost solitons, Ann. Sc. Norm. Super. Pisa Cl. Sci, (5)10(4) (2011), 757-799.

[25] Taleshian, A. and Hosseinzabeh, A. A., Investigation of Some Conditions on N(k)-Quasi Einstein Manifolds, Bull. Malays. Math. Soc. 34(3)(2011), 455-464.

[26] Gomes, J. N., Wang, Q. and Xia, C., On the h-almost Ricci soliton, J. Geom. Phys., 114 (2017), 216-222.

[27] Wang, Y. and Liu, X., Ricci solitons on three-dimensional $\eta$-Einstein almost Kenmotsu manifolds, Taiwanese Journal of Mathematics, 19(1) (2015), 91-100.

[28] Wang, Y., Ricci solitons on 3-dimensional cosympletic manifolds, Math. Slovaca, 67(4) (2017), 979-984. 